\title{
Design and efficacy of a head-coil bite bar for reducing movement-related artifacts during functional MRI scanning
}

\author{
V. MENON and K. O. LIM \\ Stanford University School of Medicine, Stanford, California \\ and Veterans Affairs Palo Alto Health Care System, Palo Alto, California \\ J. H. ANDERSON \\ Veterans Affairs Palo Alto Health Care System, Palo Alto, Califormia \\ and \\ J. JOHNSON and A. PFEFFERBAUM \\ Stanford University School of Medicine, Stanford, Califormia \\ and Veterans Affairs Palo Alto Health Care System, Palo Alto, California
}

\begin{abstract}
This article describes the design, construction, application, and effectiveness of a simple bite bar for use with an MRI whole-head coil to reduce head-movement-related artifacts during functional brain imaging. The device is comfortable to use and allows considerable flexibility in positioning the subject's head. Eleven subjects were scanned while performing a motor-sequencing experiment with and without the bite bar. The bite bar was generally effective in reducing maximum head movement to less than $0.5 \mathrm{~mm}$ (translation) and $0.5^{\circ}$ (rotation).
\end{abstract}

Functional magnetic resonance imaging (fMRI) is being increasingly used to investigate the neural substrates of perceptual and cognitive processes in the human brain (Bandettini, Wong, Hinks, \& Hyde, 1992; Blamire et al., 1992; Kwong et al., 1992; Le Bihan et al., 1995; Moseley \& Glover, 1995). Activation foci derived from functional images are overlayed on high-resolution structural images to determine brain areas involved in task-related processing. To achieve the highest accuracy possible in mapping structure to function, it is necessary to minimize all sources of movement artifact both during each imaging procedure and between structural and functional imaging. Movement during functional imaging results not only in misalignment between images, but also in fMRI signal loss. This is because the fMRI signal at a voxel is affected by the temporal history of signals at neighboring voxels (Friston, Williams, Howard, Frackowiak, \& Turner, 1996), due to the incomplete relaxation of transverse magnetic spins before the next slice is excited. While gross misalignment between images can be easily corrected, corrections for movement-related artifacts arising from signal

This research was supported in part by The Sinclair Fund, NIH (MH30854, F32BS09628, AA05965, and AGI 1427), and the Department of Veterans Affairs. The authors thank Gary Glover for fMRI data acquisition software, Margaret Rosenbloom for editorial assistance, Kurt Campbell and Kris Morrow for help with Figure 1, Douglas Schwandt for help with Figures 2 and 3, and Chuck Revell for help with Figure 4. Correspondence should be directed to V. Menon, Department of Psychiatry and Behavioral Sciences, Stanford University School of Medicine, Stanford, CA 94305 (e-mail: menon@leland.stanford.edu). loss due to altered spin history are extremely difficult to achieve, if not impossible. For these reasons, it is imperative to reduce head movement during fMRI to an absolute minimum. This consideration is particularly impor-

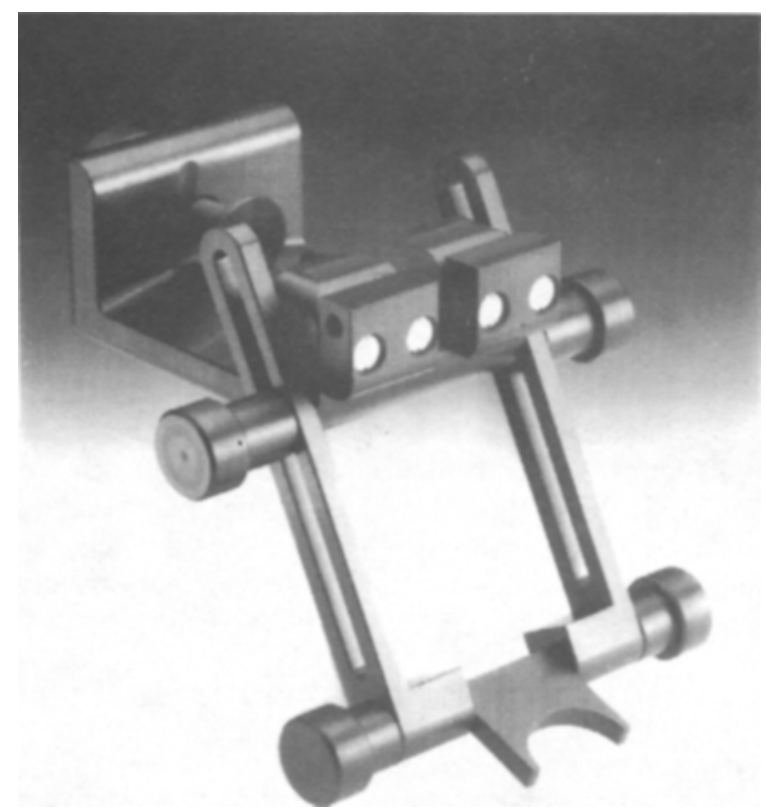

Figure 1. Side view of bite-bar holder showing $U$-shaped frame with two L-shaped slotted mounting bars at tached to it by means of thumb screws. The bite bar is screwed to the lower end of the L-shaped slotted mounting bars. 


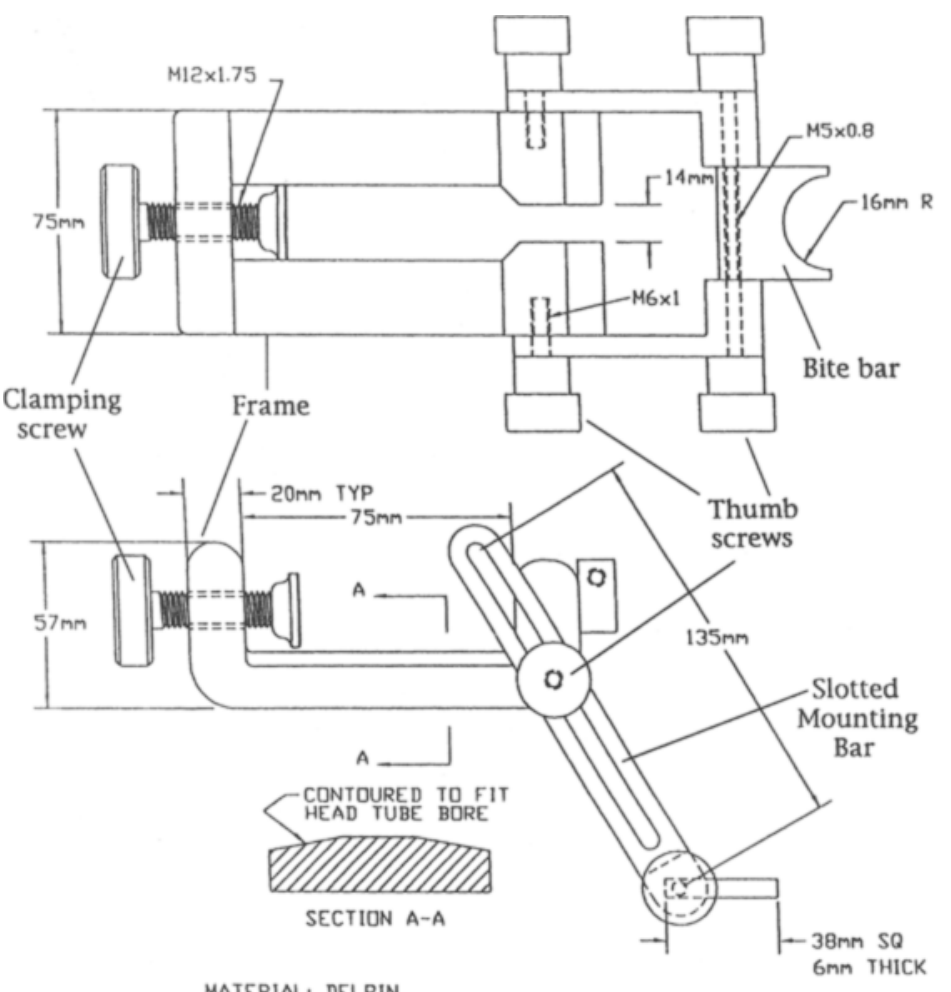

Top View

Side View

Figure 2. Details of the construction of the bite-bar holder. Top: View from the top showing frame with a metric $12 \times 1.75$ clamping screw at one end. At the other end, two slotted mounting bars are screwed with metric $6 \times 1$ thumb screws to the frame. A bite bar with semicircular cross section (radius $16 \mathrm{~mm}$ ) is screwed with two metric $5 \times 0.8$ thumb screws into the short arm of the L-shaped slotted mounting bars. Bottom: View from the side showing dimensions of the frame, $57 \mathrm{~mm}$ high $\times 75 \mathrm{~mm}$ long with 20 -mm thickness. The slotted mounting bars are $135 \mathrm{~mm}$ long. The cross-section (A-A) of the frame base is contoured to fit the head-coil bore.

tant for the studies of clinical populations that may show less inclination or capacity to lie still than do the cooperative colleagues and college students who have participated in many of the early normative fMRI studies.

Use of head coils that enable whole-brain imaging is becoming more common in fMRI protocols. This article describes the design and construction of a simple bite-bar holder for use with a standard clinical quadrature head coil (GE Medical Systems, Milwaukee). The effectiveness of the device in restricting head movement is demonstrated using functional images from 11 subjects performing a motor-sequencing task with and without the bite bar.

\section{METHOD}

\section{Design and Construction}

The design goal was to provide a comfortable bar which subjects could hold in their mouths (bite bar) and which would restrain translational and rotational displacement of the head. The bite bar is attached to a frame that is anchored nondestructively to the head coit and can be easily installed and fitted to each individual subject. All components of the bite bar and its frame were constructed out of Delrin, a nonmagnetic plastic material. The bite bar is screwed into two slotted mounting bars which are attached by screws to the frame (Figure 1). The slots in the mounting bar allow the bite bar to be raised, lowered, or rotated about the axis joining the upper thumb screws. In addition, the bite bar itself can be rotated about the axis joining the lower thumb screws.

Construction details, including component dimensions, are shown in Figure 2. The top and side views are shown in the upper and lower parts of the figure, respectively. The overall dimensions of the U-shaped frame are $50 \times 75 \times 75 \mathrm{~mm}$. At one end of the frame, a 62 -mm-long $\mathrm{Ml} 2 \times 1.75$ clamping screw is attached. The other end has a $40 \times 14 \mathrm{~mm}$ vertical slot to clear the head coil. The inner base of the frame is contoured (as shown in section A-A of Figure 2) to fit the bore of the head coil. The L-shaped slotted mounting bars, $135 \times 26 \mathrm{~mm}$ in length, are attached to the frame with two 9-mm-long M6 $\times 1$ thumb screws. Slot dimensions are $7 \times 122 \mathrm{~mm}$. A 5-mm-diam hole runs along the short leg of the "L." The overall dimensions of the bite bar are $38 \times 38 \times 6 \mathrm{~mm}$; it has a $16-\mathrm{mm}$ semicircular segment cutout at one end and an M5 $\times 0.8$ screw hole along the other end, through which two 35 -mm-long thumb screws attach the bite bar to the short leg of the mounting bars.

\section{Device Placement}

The frame of the bite-bar holder is attached to the head coil (dotted segments), as shown in Figure 3. The frame, which is held in place by the clamping screw at one end, straddles a rung that runs along the length of the head coil. The frame can be placed either under (as in Figure 3) or above the top of the head coil with equivalent fit. The two slotted mounting bars are attached to the frame with the short L-shaped segments pointing toward the center of the 


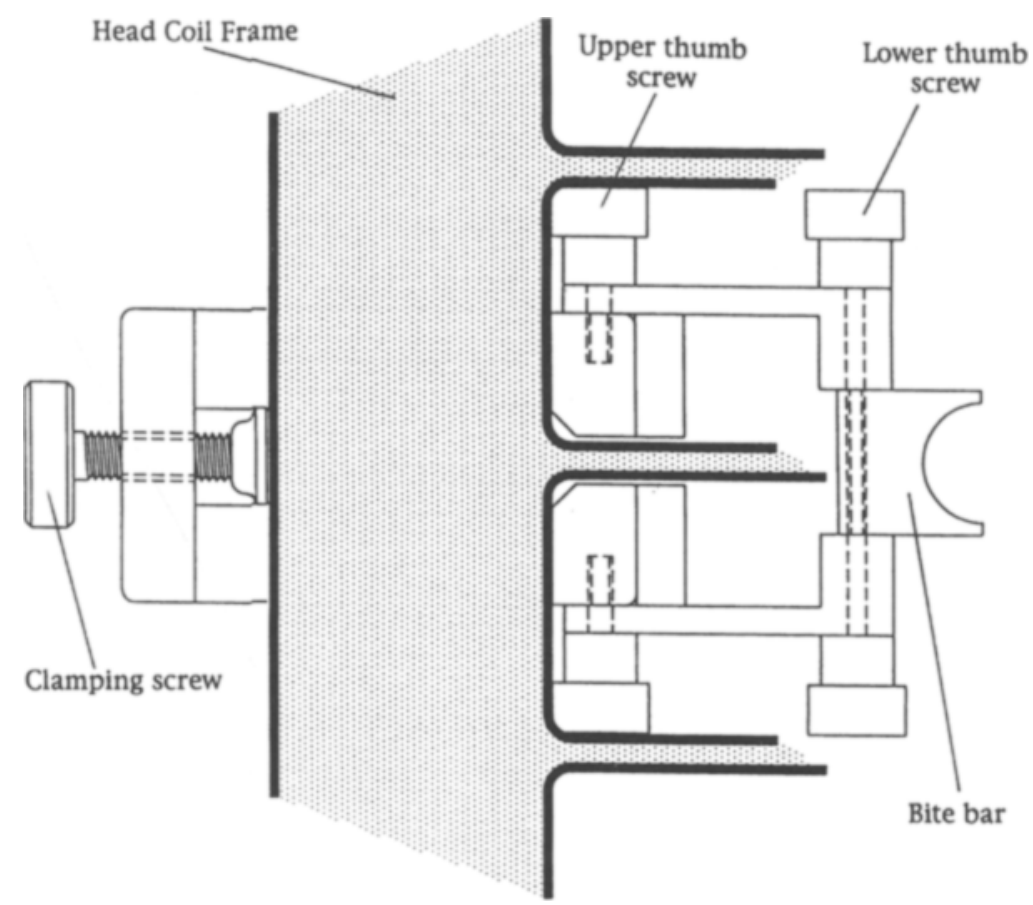

Figure 3. Top view of the bite-bar holder showing details of the way in which it is clamped to the whole head coil (dotted segment). The clamping screw (shown at left) is outside the head coil.

coil and perpendicular to the bore of the coil. The bite bar is screwed into the short L-shaped segments.

\section{Subject Preparation and Placement}

Prior to scanning, the subject's dental impression is made in moldable thermoplastic cement (Tak Hydroplastic, Wareham, MA). The whole procedure takes about $5 \mathrm{~min}$. A tablespoon of cement granules is converted to a moldable paste by placing it in a glass of warm water $\left(80^{\circ} \mathrm{C}\right)$ for $1 \mathrm{~min}$ and is then coated onto the open end of the bite bar. The subject then bites onto the bar for $30 \mathrm{sec}$, creating an impression of his/her upper and lower teeth on the cement. Once the cement has hardened $(2 \mathrm{~min})$, the bite bar, with the subject's dental impression, is then attached to the lower end of the slotted mounting bars as described above (Figure 4 , left). The subject lies supine on the scanner table and the head coil is pulled over the subject's head after the bite bar is raised to provide sufficient clearance. The bite bar is then lowered to a level such that the subject can comfortably bite into it. The bite bar and the slotted mounting bars are screwed in place using the four thumb screws (Figure 4, right).

\section{Scans}

Eleven healthy right-handed subjects ( 5 males and 6 females; ages 20-35) participated in the study after giving written informed consent. A subject lay supine in the scanner and performed the functional imaging protocol under two conditions: with the bite bar (bite-bar condition) and without the bite bar (no-bite-bar condition). While no padding was placed around the subject's head in either condition, some head support was provided by the auditory headset. Six subjects performed the protocol first with and later without the bite bar. The order of the conditions was reversed for the remaining 5 subjects. Prior to each functional scan, subjects were landmarked and structural images were acquired to localize the anterior commissure (AC).

Structural and functional images were acquired on a conventional 1.5T GE scanner using a quadrature head coil. T2-weighted structural images were acquired in the sagittal plane with a slice thick- ness of $3 \mathrm{~mm}$. Fifteen slices centered in the midsagittal plane were acquired. A fast-spin echo-pulse sequence with the following parameters was used for this purpose: $\mathrm{TR}=2,500 \mathrm{msec}$, TE $=85 \mathrm{msec}$, and echo-train length $=8$. These images were used to localize the $\mathrm{AC}$ and other neuroanatomical landmarks. Functional images were then acquired using a T2*-weighted gradient echo spiral pulse sequence with the following parameters: TR $=67 \mathrm{msec}, T E=40 \mathrm{msec}$, flip angle $=40^{\circ}$, and 4 interleaves (Moseley \& Glover, 1995). Twelve axial slices were acquired roughly between $-30 \mathrm{~mm}$ below the $\mathrm{AC}$ and $54 \mathrm{~mm}$ above the $\mathrm{AC}$, with in-plane spatial resolution of $1.21 \mathrm{~mm}, 6-\mathrm{mm}$ slice thickness, $1-\mathrm{mm}$ interslice thickness, and a $310-\mathrm{mm}$ field of view. The sampling time, the time taken to acquire 12 slices, was $4 \mathrm{sec}$. Each functional imaging protocol had 120 frames and took $8 \mathrm{~min}$.

Table 1

Mean \pm Standard Deviation of the Maximum Translational and Rotational Displacement, Across 11 Subjects, Along the Three Axes With (Bite-Bar Condition) and Without (No-Bite-Bar Condition) the Bite Bar

\begin{tabular}{|c|c|c|c|c|c|c|}
\hline \multirow[b]{2}{*}{ Axis } & \multicolumn{2}{|c|}{ No Bite Bar } & \multicolumn{2}{|c|}{ Bite Bar } & \multirow[b]{2}{*}{$t$} & \multirow[b]{2}{*}{$p$} \\
\hline & $M$ & $S D$ & $M$ & $S D$ & & \\
\hline \multicolumn{7}{|c|}{ Translation } \\
\hline$x(\mathrm{~mm})$ & 0.73 & 0.74 & 0.38 & 0.20 & 1.4218966 & .093 \\
\hline$y(\mathrm{~mm})$ & 0.55 & 0.49 & 0.23 & 0.12 & 2.3854015 & .019 \\
\hline$z(\mathrm{~mm})$ & 1.36 & 1.01 & 0.35 & 0.23 & 3.1196249 & .005 \\
\hline \multicolumn{7}{|c|}{ Rotation } \\
\hline$x$ (degrees) & 1.09 & 0.78 & 0.19 & 0.09 & 3.7998606 & .002 \\
\hline$v$ (degrees) & 0.63 & 0.51 & 0.31 & 0.13 & 1.9222660 & .042 \\
\hline$=$ (degrees) & 0.51 & 0.25 & 0.31 & 0.20 & 1.8709000 & .045 \\
\hline
\end{tabular}

Note-The $t$ statistic and one-tailed paired $t$ test $p$ value for differences between the conditions indicates that use of the bite bar significantly reduced movement. 

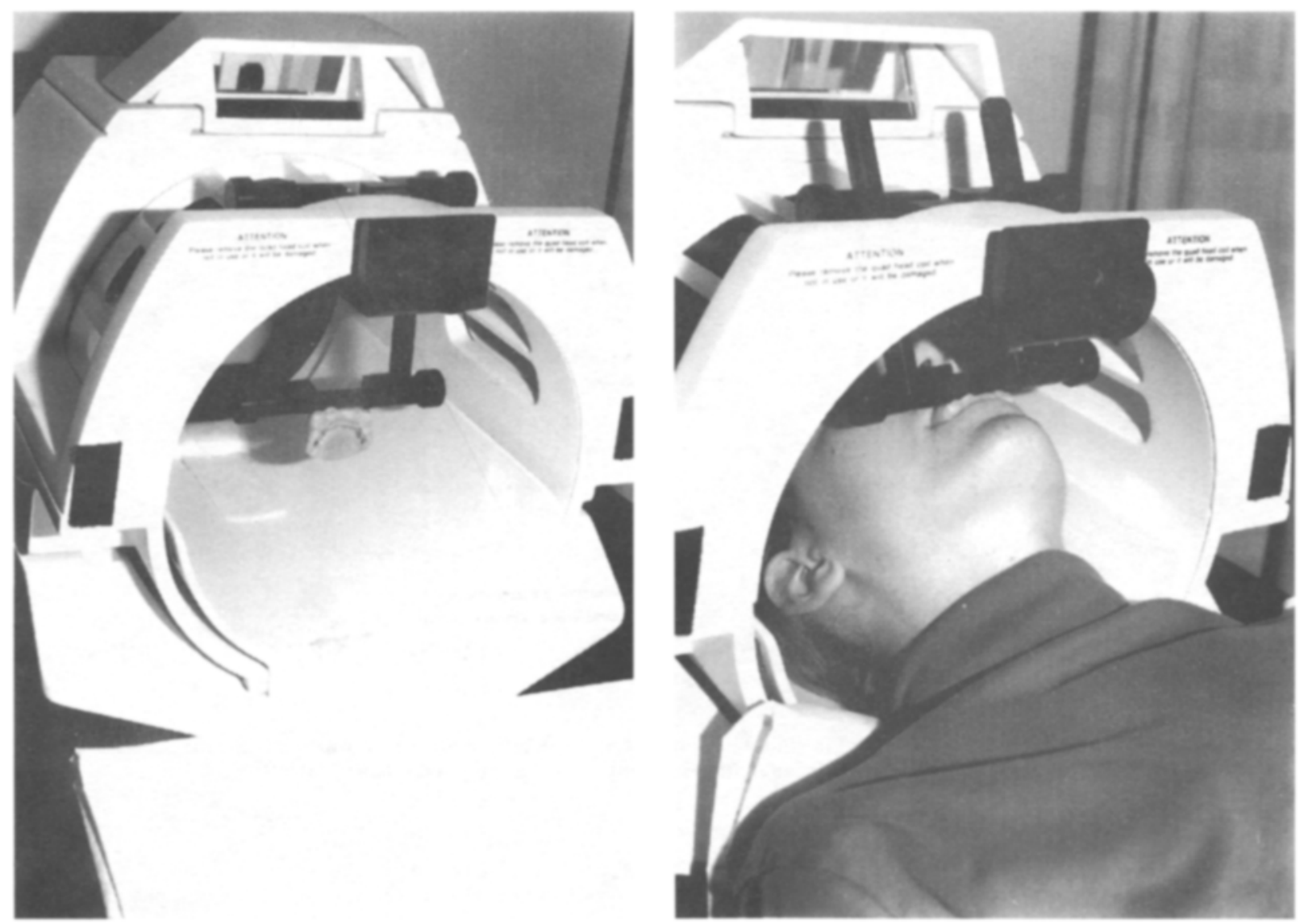

Figure 4. Left: The bite-bar holder clamped to the head coil. The bite bar with the subject's dental impression is attached to the frame of the holder with the two slotted mounting bars. Right: View of a subject biting on bar in the head coil.

The functional imaging protocol consisted of 12 alternating 40 sec epochs of rest and a motor task. During the motor task, a subject heard randomly generated numbers between 1 and 4 with an interstimulus interval of $3 \mathrm{sec}$. The duration of each stimulus was $250 \mathrm{msec}$. The subject made arm movements to one of four corresponding locations on a keypad, strapped to his/her thigh, starting from a rest position at the base of the keypad. The distance from the base of the keypad to each of the keys was $15 \mathrm{~cm}$. All subjects made movements with their right hands.

The task was programmed using Psyscope (Cohen, MacWhinney, Flatt, \& Provost, 1993) on a Macintosh notebook computer. Audio signals from the Macintosh were amplified using a standard home audio receiver and transmitted to a piezo-electric speaker placed near the head of the scanner. Sound was piped binaurally to the subject by means of a plastic headset connected with a plastic tube to a funnel placed over the piezo-electric speaker. Start of the scanning and task was synchronized using a TTL pulse delivered to the scanner timing microprocessor board using a "CMU Button Box" microprocessor (http://poppy.psy.cmu.edu/psyfiles/bbox) connected to the Macintosh with a serial cable.

For each functional scan, a raw binary file, containing data for 120 time points and 12 slices, was written to computer disk.

\section{Analysis}

fMRI images were reconstructed from the binary files and analyzed for movement-related displacement as a function of time. Image files were constructed for each of the 120 time points, with each image file containing data for the 12 slices. The first two image files were discarded to reduce the nonequilibrium effects of scan onset. Translation and rotation along the three orthogonal axes $(x, y$, and $z)$ were assessed by realigning each of the last 117 images with respect to the third image. Images were realigned using a leastsquares minimization algorithm based on the differences between images (Snyder, 1995), as implemented in the Statistical Parametric Mapping software (Friston et al., 1995). No corrections for spin history were made.

The maximum absolute displacements (translation and rotation) in each of the three axes were calculated for each subject in each condition (bite bar vs. no bite bar). This metric rather than the mean and standard deviation of the time series was used because the observations are not truly independent. In particular, the mean is not useful because of the possibility of large positive and negative displacements about zero. Mean and standard deviation of the maximum displacements in each axis were calculated across the 11 subjects. One-tailed paired $t$ tests were used to determine whether the maximum displacements were significantly reduced by using the bite bar.

The $x$-axis was aligned from the left side of the head to the right, the $y$-axis was aligned from the back of the head to the front, and the $z$-axis was aligned from the bottom of the head to the top.

\section{RESULTS}

The task resulted in significant activation of a number of sites in the basal ganglia-thalamocortical loop (see, 
$\mathbf{x}$
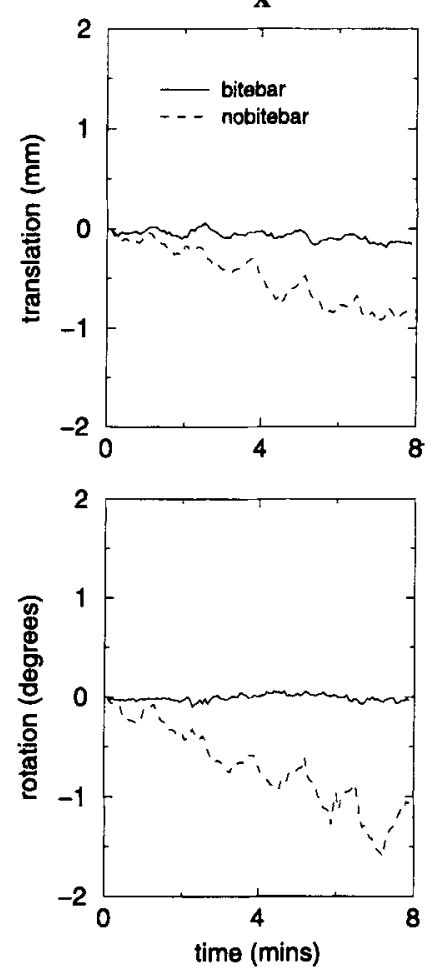
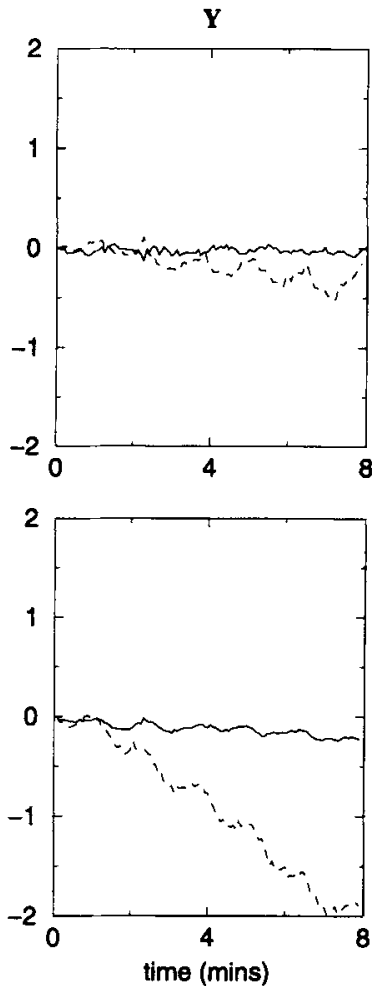

$\mathbf{z}$
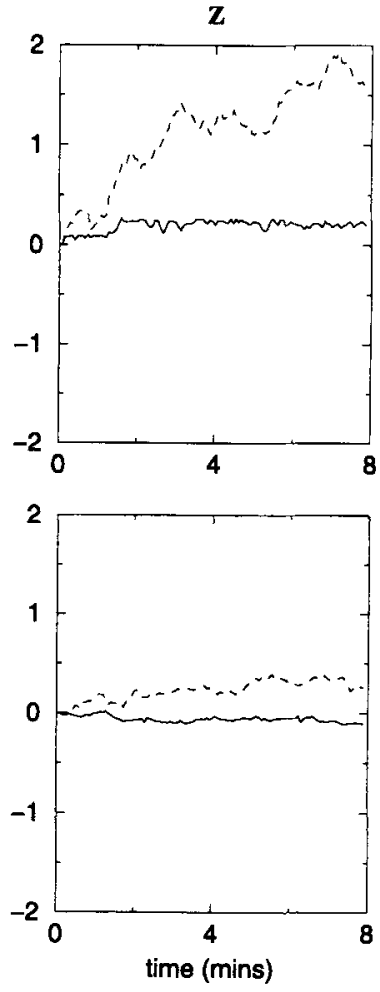

Figure 5. Displacement of functional MR images, with (solid line) and without (dashed line) a bite bar, as a function of scan time during a motor-sequencing experiment. $x, y$, and $z$ components of the translation (top row) and rotation (bottom row) are shown. Image displacements are considerably reduced when the bite bar is used. The $x$-axis is aligned from the left side of the head to the right, the $y$-axis is aligned from the back of the head to the front, and the $z$-axis is aligned from the bottom of the head to the top.

e.g., Menon et al., 1996). For this report, we focus on the comparison of movement-related displacement of images under the bite-bar and no-bite-bar conditions. Figure 5 shows the translation and rotation needed to realign the last 117 images with respect to the third image for each condition for the subject identified as number 3 in Figure 6. The displacements have a steady drift with random fluctuations superimposed on it. Without the bite bar, large translational drifts along the $z$-axis and rotational drifts about the $x$-axis were observed in most subjects. In all 11 subjects, use of a bite bar reduced maximum translation along and rotation about the $x-, y=$, and $z$-axes (Figure 6). Table 1 shows the mean $\pm S D$, across 11 subjects, of the maximum translation along and rotation about the three axes for each condition. The $t$-test statistic and one-tailed paired $p$ value for condition differences between the maximum translation and rotation for each axis are also shown. Reductions in displacement due to use of the bite bar were statistically significant $(p<.05)$ in all directions (except translation along the $x$-axis, where displacements without the bite bar were small to begin with). The largest magnitude of reductions was observed for translation along the $z$-axis and rotation about the $x$-axis. These reductions were highly statistically significant $(p<.005)$.

\section{DISCUSSION}

The bite bar was effective in reducing the maximum translation along the $x-, y$-, and $z$-axes to less than $0.5 \mathrm{~mm}$ and the maximum rotation about the $x$-, $y$-, and $z$-axes to less than $0.5^{\circ}$ in most of the subjects (Table 1 ). Without the bite bar, the largest translational drifts were observed along the $z$-axis $(1-3 \mathrm{~mm}$ ) and the largest rotational drifts were observed about the $x$-axis $\left(0.5^{\circ}-2^{\circ}\right)$. The bite bar was particularly effective in reducing these displacements. Translation along the $x$-axis was relatively small in the no-bite-bar condition due possibly to the padding provided by the headset used to pipe in the auditory stimuli. However, this was not sufficient to prevent rotation about the $x$-axis (i.e., in the sagittal plane). The standard deviation across subjects decreased more with the bite-bar than with the no-bite-bar condition. This indicates that the bite bar reduces variability in the motion across subjects.

Restraining head movement not only reduces such gross movement-related artifacts as spatial blurring, but may also allow for the detection of signals that might otherwise be lost due to movement-related changes in spin history. Use of such a device may improve the quality of $f M R I$ data and help comparison of functional activation studies across laboratories. 
$\mathbf{x}$
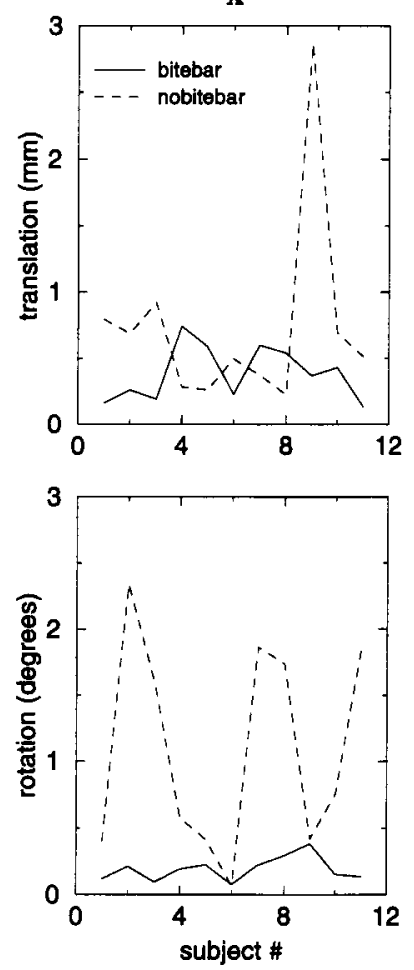

$\mathbf{Y}$
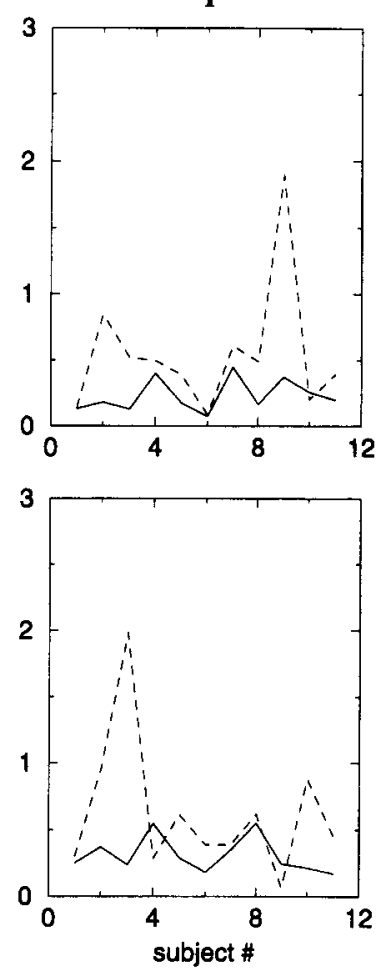

$\mathbf{Z}$
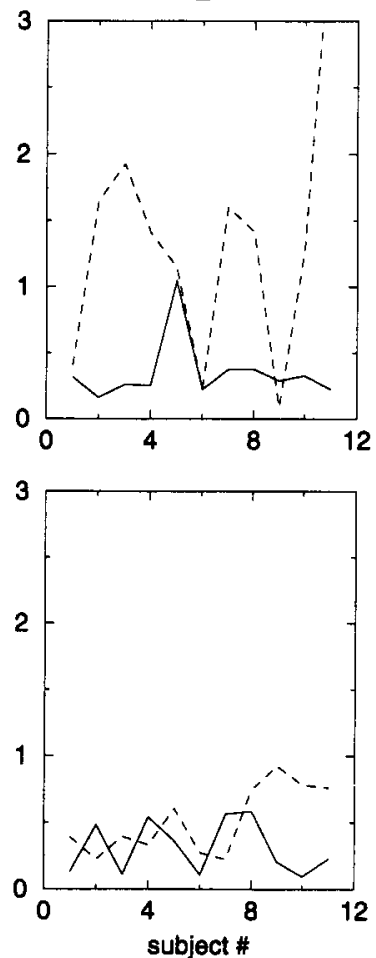

Figure 6. Maximum displacement of functional MRI images, with (solid line) and without (dashed line) a bite bar, across subjects during a motor-sequencing experiment. $x, y$, and $z$ components of the translation (top row) and rotation (bottom row) are shown. In most of the subjects, maximum displacements are reduced to less than $0.5 \mathrm{~mm}$ (translation) and $0.5^{\circ}$ (rotation) when the bite bar is used. Note the large translational displacements along the $z$-axis and rotational displacements along the $x$-axis without the bite bar.

The bite-bar device allowed considerable flexibility and reasonable comfort in positioning and restraining the subject's head. It has been used with 30 normal subjects in six different experiments; no subjects complained of discomfort, and all tolerated the device well. Estimated cost of construction is about $\$ 500$, which can be recouped by avoiding the loss of data from one acquisition session at our current cost for scanner time.

One possible alternative to the bite bar is padding around the subject's head. However, as the above results suggest, this may not be sufficient to prevent rotational displacement (about the $x$-axis) or drift along the axis parallel to the scanner bore ( $z$-axis). One possible disadvantage of the bite bar relates to complications in paradigms involving speech.

\section{REFERENCES}

Bandettini, P. A., Wong, E. C., Hinks, R. S., \& Hyde, J. S. (1992). Time course EPI of human brain function during task activation. Magnetic Resonance in Medicine, 25, 390-397.

Blamire, a. M., Ogawa, S., Ugurbil, K., Rothman, D., McCarthy, G., Ellermann, J. M., Hyder, F., Rattner, Z., \& ShulMAN, R. G. (1992). Dynamic mapping of the human visual cortex by high-speed magnetic resonance imaging. Proceedings of the $\mathrm{Na}$ tional Academy of Sciences, 89, $11069-11073$.

Cohen, J., MacWhinney, B., Flatt, M., \& Provost, J. (1993). PsyScope: An interactive graphic system for designing and controlling experiments in the psychology laboratory using Macintosh computers. Behavior Research Methods, Instruments, \& Computers, 25, 257-271. Friston, K. J., Holmes, A. P., Worsley, K. J., Poline, J. P., Frith, C. D., \& FRACKOWIAK, R. S. J. (1995). Statistical parametric maps in functional imaging: A general linear approach. Human Brain Mapping, 2, 189-210.

Friston, K., Williams, S. R., Howard, R., Frackowiak, R. S. J., \& TURNER, R. (1996). Movement-related effects in fMRI time-series. Magnetic Resonance in Medicine, 35, 346-355.

Kwong, K. K., Belliveau, J. W., Chesler, D. A., Goldberg, I. E., Weisskoff, R. M., Poncelet, B. P., Kennedy, D. N., HopPel, B. E., Cohen, M. S., \& Turner, R. (1992). Dynamic magnetic resonance imaging of human brain activity during primary sensory stimulation. Proceedings of the National Academy of Sciences, 89, 5675-5679.

Le Bihan, D., Jezzard, P., Haxby, J., Sadato, N., Rueckert, L., \& MATTAY, V. (1995). Functional magnetic resonance imaging of the brain. Annals of Internal Medicine, 122, 296-303.

Menon, V., Johnson, J., Sullivan, E. V., Lim, K. O., Glover, G. H., \& Pfefferbaum, A. (1996). Functional MRI activation of multiple basal ganglia-thalamocortical sites during self-generated sequences of arm movements. Society for Neuroscience Abstracts, 22, 1085.

Moseley, M. E., \& Glover, G. H. (1995). Functional MR imaging: Capabilities and limitations. Neuroimaging Clinics of North America, 5, 161-191.

SNYDER, A. Z. (1995). Difference image vs. ratio image error function forms in PET-PET realignment. In R. Myer, V. J. Cunningham, D. L. Bailey, \& T. Jones (Eds.), Quantification of brain function using PET (pp. 131-137). San Diego: Academic Press.

(Manuscript received January 23, 1996; revision accepted for publication December $6,1996$. 